\title{
A Wide-Band Slot Antenna Design Employing A Fictitious Short Circuit Concept
}

\author{
Nader Behdad, Student Member, IEEE, and Kamal Sarabandi, Fellow, IEEE
}

\begin{abstract}
A wide-band slot antenna element is proposed as a building block for designing single- or multi-element wide-band or dual-band slot antennas. It is shown that a properly designed, offcentered, microstrip-fed, moderately wide slot antenna shows dual resonant behavior with similar radiation characteristics at both resonant frequencies and therefore can be used as a wide-band or dual-band element. This element shows bandwidth values up to $37 \%$, if used in the wide-band mode. When used in the dual-band mode, frequency ratios up to 1.6 with bandwidths larger than $10 \%$ at both frequency bands can be achieved without putting any constraints on the impedance matching, cross polarization levels, or radiation patterns of the antenna. The proposed wide-band slot element can also be incorporated in a multi-element antenna topology resulting in a very wide-band antenna with a minimum number of elements. It is also shown that, by using only two of these elements in a parallel feed topology, an antenna with good radiation parameters over a 2.5:1 bandwidth ratio can be obtained.
\end{abstract}

Index Terms-Broad-band antennas, multiple band antennas, slot antennas.

\section{INTRODUCTION}

W IDE BANDWIDTH, small size, ease of fabrication, low cost, and compatibility to the rest of the RF front end are desirable factors of an antenna. The literature concerning the design of frequency independent or very wide-band antennas is enormous and extends beyond the scope of this paper. The vast majority of techniques developed for achieving frequency independent behavior make use of topologies that result in relatively large antennas, hence eliminating their use for mobile wireless applications. Therefore, it is desirable to develop other techniques to increase the bandwidth of, otherwise narrow-band, small-sized antennas, without significantly increasing their sizes. This can be accomplished by increasing the number of resonances of single resonant antennas such as a half-wavelength slot. For example, by feeding a $\lambda / 2$ slot near an edge by a microstrip line, the magnetic current distribution (electric field along the slot) can be manipulated to create a null near the feed point which produces a second resonance with a frequency slightly larger than that of the first one. When appropriately fed, these two resonances can merge and result in an antenna with a much larger bandwidth or two separate bands of operation with similar radiation characteristics. Microstrip-fed, wide slot antennas have been theoretically studied in [1]. Also

Manuscript received November 12, 2003; revised May 17, 2004. This work was supported in part by the Engineering Research Centers Program of the National Science Foundation (NSF) under Grant EEC-9986866.

The authors are with the Radiation Labratory, Department of Electrical Engineering and Computer Science, The University of Michigan, Ann Arbor, MI 48109-2122 USA (e-mail: behdad@engin.umich.edu)

Digital Object Identifier 10.1109/TAP.2004.838778 experimental investigations on very wide slot antennas are reported by various authors [2]-[5]. The drawback of these antennas are two fold: 1) they require a large area for the slot and a much larger area for the conductor plane around the slot and 2) the cross polarization level changes versus frequency and is high at certain frequencies in the band [2]-[4], and [6]. This is mainly because of the fact that these antennas can support two orthogonal modes with close resonant frequencies. The advantages of the proposed dual-resonant slots to these antennas are their simple feeding scheme, low cross polarization levels, and amenability of the elements' architecture to the design of series-fed multi-element broad-band antennas. That is, the proposed wide-band element can be used as a building block in design of multi-element antennas that can produce larger overall bandwidths with fewer radiating elements and much smaller size than existing multi-element antennas such as log-periodic arrays. In this paper, first the design and measurement results of the double-resonant slot antenna in the wide-band mode is presented. Then the application of this technique in the design of dual-band slot antennas is examined, and finally the use of this element in a parallel fed double-element antenna topology is investigated. The latter antenna shows very good radiation characteristics over a $2.5: 1$ bandwidth ratio by using only two radiating elements.

\section{OfF-Centered Microstrip-Fed Slot Antenna AS A BROAD-BAND ELEMENT}

\section{A. Antenna Design}

A resonant narrow slot antenna is equivalent to a magnetic dipole, and at its first resonant frequency it has an equivalent length of $\lambda_{g} / 2$ ( $\lambda_{g}$ is the guided wavelength in the slot). If the slot antenna is fed near an edge by a microstrip line and the slot width is properly chosen, at a frequency above the first slot resonance, a fictitious short circuit near the microstrip feed may be created. Basically the tangential component of the electric field created by the microstrip line at a particular distance cancels out the electric field of the slot excited by the return current on the ground plane of the microstrip line. A full wave simulation shows the field distribution for this situation in Fig. 1(b) whereas Fig. 1(a) shows the electric field distribution in the slot at its normal first resonance. The field distribution is shown to have a null along the slot between the two ends at a frequency slightly above the first resonance. The width of the microstrip feed, the slot width, and the distance between the center of the microstrip feed and the edge of the slot antenna $L_{s}$, are the parameters that affect the existence and location of this fictitious short circuit. As $L_{s}$ increases, the second resonant frequency also in- 


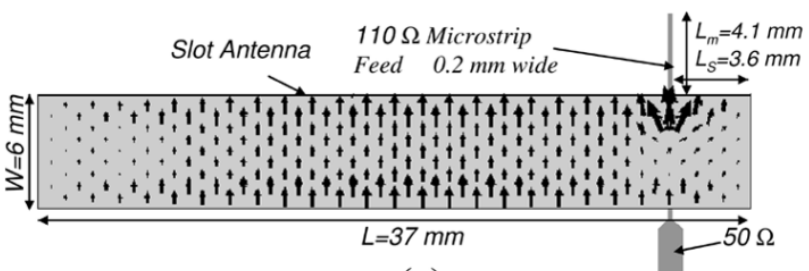

(a)

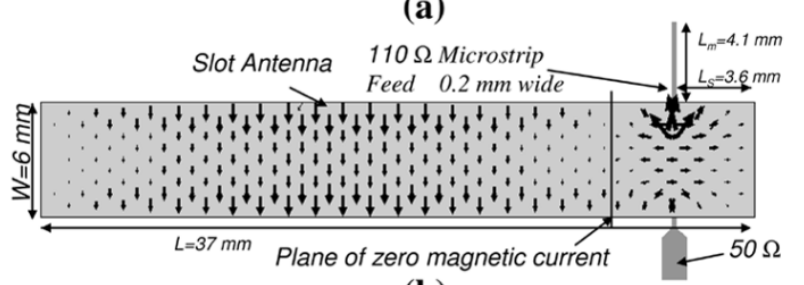

(b)

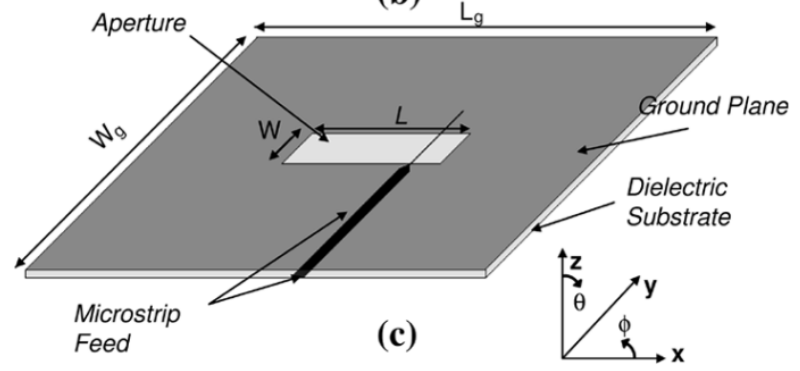

Fig. 1. Electric field distributions and three-dimensional geometry of a $0.08 \lambda$ wide microstrip-fed slot antenna. (a) Normal field distribution. (b) Field distribution at a slightly higher frequency showing a fictitious short circuit along the slot causing the second resonance. (c) Three-dimensional geometry.

creases; therefore by choosing $L_{s}$ properly, the second resonant frequency can be chosen such that the total antenna bandwidth is increased or a dual-band operation is achieved. Meanwhile the frequency of the first resonance, which is determined by the overall effective length of the antenna, does not change significantly. Matching is achieved by tuning the length of the open circuited microstrip line $L_{m}$. As can be observed from Fig. 1, the electric field distribution at this second resonant frequency is similar to that of the first one. Therefore, it is expected that the radiation patterns of the antenna at the two frequencies are similar.

In order to test this concept, two wide-band double-resonant slot antennas with different $L_{s}$ values are designed and fabricated. The antennas [Fig. 1(c)] are similar rectangular slots with lengths and widths of $L=37 \mathrm{~mm}$ and $W=6 \mathrm{~mm}$ and $L_{s}$ of 3.6 and $4.6 \mathrm{~mm}$ for Antenna 1 and Antenna 2, respectively. The antennas are fed with a $110 \Omega$ open circuited microstrip-line connected to a $50 \Omega$ main line that is connected to a $50 \Omega$ SMA connector. The structures were simulated using IE3D [8] and fabricated on a $500 \mu \mathrm{m}, \mathrm{RO} 4350 \mathrm{~B}$ substrate with a dielectric constant of 3.4 , loss tangent of 0.003 , and ground plane size of $L_{g} \times W_{g}=15 \mathrm{~cm} \times 12 \mathrm{~cm}$. Fig. 2 shows the simulated and measured input reflection coefficients of both antennas and Table I shows a summary of radiation parameters and physical dimensions of the two antennas. The $-10 \mathrm{~dB} S_{11}$ bandwidth of Antenna 1 is $1022 \mathrm{MHz}(30.3 \%)$, whereas Antenna 2 shows a bandwidth of $1220 \mathrm{MHz}(37 \%)$. The slight discrepancy between the simulation and measurement results can be attributed to the finiteness of the ground plane, which causes a shift in the

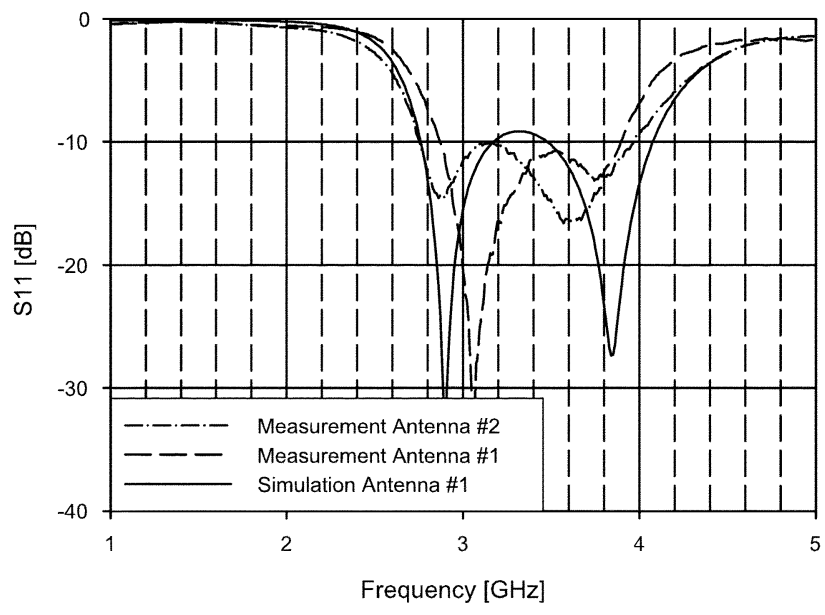

Fig. 2. Measured and simulated input reflection coefficients of the proposed broad-band slot antenna. The two measured responses correspond to two values of $L_{S}=3.6 \mathrm{~mm}$ and $L_{S}=4.6 \mathrm{~mm}$.

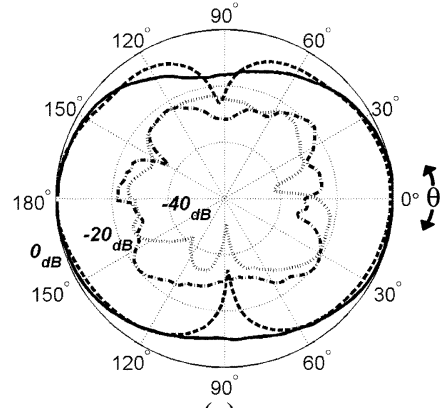

(a)

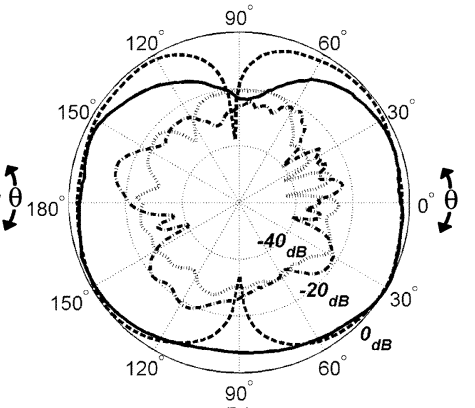

(b)
Fig. 3. E- and H-plane co- and cross-polarized radiation patterns of the broadband slot antenna. "-": H-plane Co-pol, “- .": E-plane Co-pol, “-.”: H-plane X-pol, and “...”: E-plane X-pol.

resonant frequency and to the fact that the response of the antenna is sensitive to the exact location of the microstrip feed, which is subject to alignment errors in the fabrication process. Radiation patterns of Antenna 1 were measured in the anechoic chamber of the University of Michigan at the two resonant frequencies. Fig. 3(a) and (b) show the E- and H-plane co- and cross-polarized patterns of this antenna at 3.077 and $3.790 \mathrm{GHz}$, respectively. It is observed that the patterns at both resonant frequencies are similar, confirming the fact that electric field distributions along the slot are similar at both frequencies. As can be observed from this figure, the E-plane pattern of the antenna is not the dual of those of an electric dipole. From duality theorem, it is expected that the E-plane patterns are similar to the $\mathrm{H}$-plane pattern of an electric dipole, which is a circle. However, in Fig. 3 the E-plane patterns have two minima at $\theta= \pm 90^{\circ}$. This is caused by two different mechanisms. The first mechanism is the cancellation that occurs at grazing angle as a result of the phase difference between E-fields on the top and bottom of the ground plane. The second reason for having these minima is that the slot antenna is covered with a dielectric substrate at one side. This forces the normal component of the electric field at grazing angle to go to zero as described in [7]. It is also observed that the normalized cross polarization level at broadside 
TABLE I

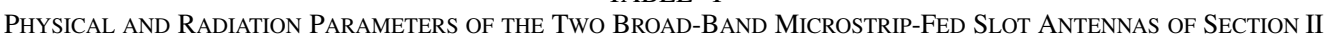

\begin{tabular}{c||c|c|c|c|c}
\hline Type & $L, W$ & $L_{s}, L_{m}$ & Bandwidth & Gain @ $f_{l}, f_{u}$ & $L_{g}, W_{g}$ \\
\hline Antenna 1 & $37 \mathrm{~mm}, 6 \mathrm{~mm}$ & $3.6 \mathrm{~mm}, 4.1 \mathrm{~mm}$ & $30.3 \%$ & $2.5 \mathrm{~dB}, 2.6 \mathrm{~dB}$ & $15 \mathrm{~cm}, 12 \mathrm{~cm}$ \\
\hline Antenna 2 & $37 \mathrm{~mm}, 6 \mathrm{~mm}$ & $4.6 \mathrm{~mm}, 3.9 \mathrm{~mm}$ & $37 \%$ & $2.5 \mathrm{~dB}, 2.5 \mathrm{~dB}$ & $15 \mathrm{~cm}, 12 \mathrm{~cm}$ \\
\hline
\end{tabular}

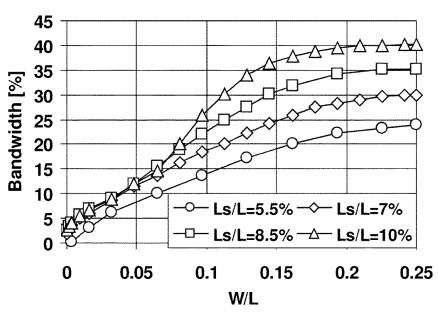

(a)

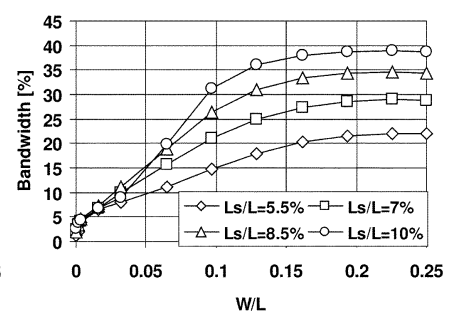

(b)

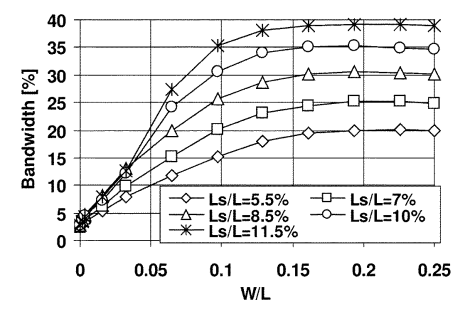

(c)

Fig. 4. Bandwidth of moderately-wide slot antenna in the wide-band mode of operation as a function of aspect ratio $(W / L)$, feed impedance, and location of the feed. (a) $50 \Omega$ feed impedance, (b) $80 \Omega$ feed impedance, and (c) $110 \Omega$ feed impedance.

is less than $-25 \mathrm{~dB}$ and small for other observation angles as well. The gains of the antennas were measured in the anechoic chamber using a standard double ridged horn antenna at the two different resonant frequencies and are reported in Table I. The antenna gains at both frequencies are very similar which again assures the similarity of the radiation mechanisms and the field distributions at both frequency bands.

\section{B. Parametric Study}

Now that the broad-band behavior of the microstrip-fed slot antenna is shown, the effect of the three parameters that affect its double-resonant behavior must also be examined. These parameters are the impedance of that section of microstrip line that feeds the slot antenna, the aspect ratio of the antenna $(W / L)$, and the distance between the edge of the slot antenna and the center of the microstrip feed $L_{s}$. Using full-wave simulations in IE3D, a number of slot antennas with different $W / L, L_{s}$, and feed impedance values are designed and matched to $50 \Omega$ by changing the length of the open circuited microstrip line $\left(L_{m}\right.$ in Fig. 1). Then their impedance bandwidths $\left(\left|S_{11}\right|<-10 \mathrm{~dB}\right)$ are calculated and reported in Fig. 4. The antennas have aspect ratios $(W / L)$ ranging from 0.0003 to 0.25 . In this paper, the antennas that have aspect ratios in the range of $0.05<$ $W / L<0.25$ are referred to as "moderately-wide" and those with $W / L<0.05$ and $W / L>0.25$ are referred to as "narrow" and "wide" slot antennas, respectively. Fig. 4(a) shows the fractional bandwidth versus aspect ratios of the antennas when they are fed with $50 \Omega$ microstrip lines. From this figure, it can be observed that for a fixed $L_{s}$, as $W / L$ increases the antenna band- width increases as well. However, the increase in bandwidth for $0.2<W / L<0.25$ is not significant. This saturation behavior is explained by defining a quality factor for the antenna and relating it to the Fourier transform of the electric field over the aperture. It can be shown that for a single-resonant slot antenna bandwidth increases as $W$ increases and a similar saturation behavior is observed [9]. This can then be extended to each of the resonances of this double-resonant antenna. The doubleresonant behavior for the $50 \Omega$ line impedance is only observed for $W / L>0.07$. For $L_{s} / L=5.5 \%$, the antenna is hard to match for small $W / L$ values and hence, its impedance bandwidth goes to zero faster than other cases. It is also observed that for a fixed $W / L \geq 0.07$, antenna bandwidth increases as $L_{s}$ increases. This increase continues until $L_{s} / L=10 \%$. Beyond this value increasing $L_{s}$ increases the second resonant frequency such that a wide-band match cannot be obtained and the antenna enters into the dual-band mode of operation.

Fig. 4(b) shows a similar study in the same $W / L$ range for antennas fed with $80 \Omega$ microstrip lines. In this case, the doubleresonant behavior is observed for $W / L \geq 0.05$ and the antenna bandwidth has a similar characteristics as the previous case. Similar to the previous case, the antenna goes to dual-band mode for $L_{s} / L>10 \%$. Fig. 4(c) shows a similar figure for a $110 \Omega$ feed line. In this case, the antenna does not go to dual-band mode until $L_{s} / L>11.5 \%$. This occurs because the separation between the two resonant frequencies $\left(f_{u}-f_{l}\right)$ is smaller for the same $L_{s}$ value and matching the antenna, by only changing $L_{m}$ was found to be easier. Comparing Fig. 4(a)-(c) shows that for the same $W / L$ and $L_{s}$ values, the bandwidth of the antenna slightly decreases as the feed impedance increases. This can be attributed to the presence of the discontinuity in the transition region from the main $50 \Omega$ line to $80 \Omega$ or $110 \Omega$ feed lines. However, under the same condition, matching is easier and lower $S_{11}$ values can be obtained for higher feed impedances.

\section{Dual-BAnd MicrostriP-Fed Slot Antenna}

\section{A. Antenna Design}

In this section, we will show that the moderately-wide slot antenna presented in the previous section can also be designed to demonstrate a dual-band behavior. Referring to Fig. 1(b), if the distance $L_{s}$ is increased, the equivalent length of the second resonance decreases. Therefore the second resonant frequency $\left(f_{u}\right)$ increases while the frequency of the first resonance $\left(f_{l}\right)$ is not expected to change considerably, as this resonance is set by the overall length of the antenna. This way the antenna becomes a dual-band one in which the separation between the two resonant frequencies is a function of the resonant length of the second resonant frequency or, equivalently, the distance of the feed from the antenna edge, $L_{s}$. Fig. 5 shows the full-wave $S_{11}$ simulation results of an antenna with $W=6 \mathrm{~mm}$ and $L=31 \mathrm{~mm}$ on a 


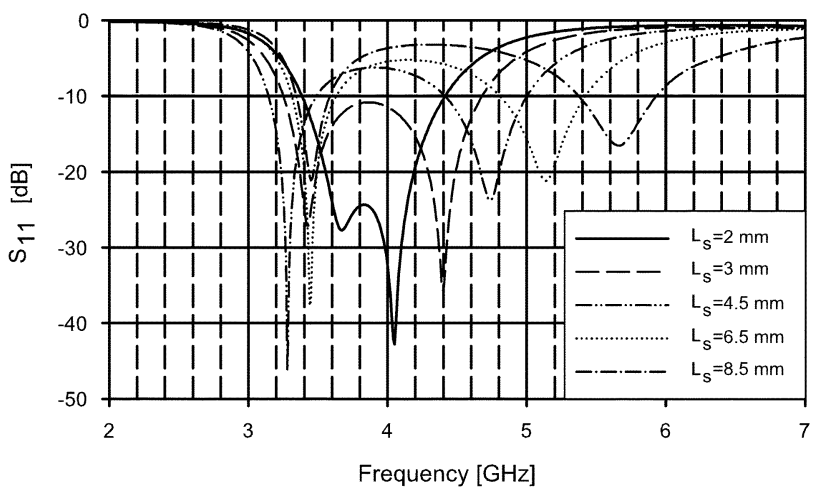

Fig. 5. Simulated input reflection coefficients of a dual-band antenna with $W=6 \mathrm{~mm}$ and $L=31 \mathrm{~mm}$ on a substrate with $\epsilon_{r}=3.4$ for different values of $L_{s}$.

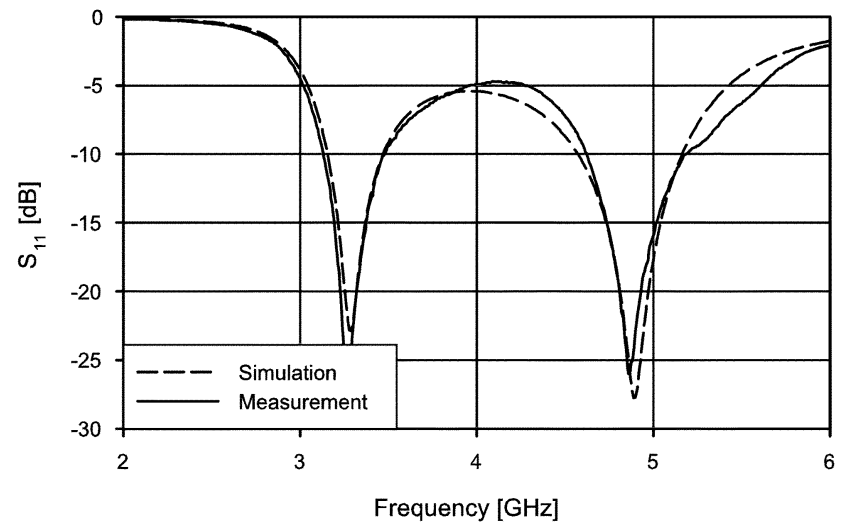

Fig. 6. Simulated and measured input reflection coefficients of the dual-band antenna of Section III.

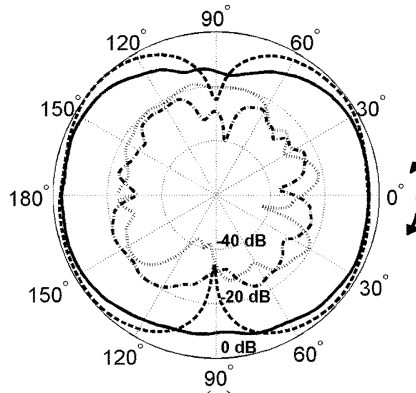

(a)

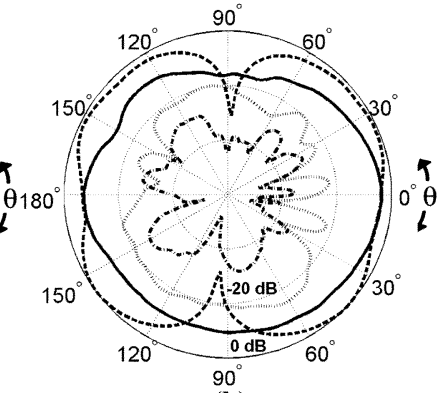

(b)
Fig. 7. E- and H-plane co- and cross-polarized radiation patterns of the dual-band slot antenna of Section III at (a) $f=3.3 \mathrm{GHz}$ and (b) $f=$ $4.85 \mathrm{GHz}$. “-”: H-plane co-pol, “- -": E-plane co-pol, “-.”: H-plane X-pol, and “...": E-plane X-pol.

substrate with dielectric constant of 3.4 , for different values of $L_{s}$. It is seen that by increasing $L_{s}$ from 2 to $8.5 \mathrm{~mm}, f_{l}$ varies between 3.3 and $3.7 \mathrm{GHz}$ and $f_{u}$ increases from 4 to $5.2 \mathrm{GHz}$. This corresponds to an $f_{u} / f_{l}$ variation from 1.1 to 1.6 while retaining an $S_{11}$ lower than $-15 \mathrm{~dB}$.

Based on the simulation results of Fig. 5, a dual-band antenna with $L=31 \mathrm{~mm}$ and $W=6 \mathrm{~mm}$ was designed and fabricated on a $500 \mu \mathrm{m}$ thick, RO4350B substrate with a ground plane size of $15 \mathrm{~cm} \times 12 \mathrm{~cm}$. The simulated and measured $S_{11}$ of this antenna are plotted in Fig. 6, where excellent agreement between them is observed. The antenna shows a $f_{u} / f_{l}$ of 1.47 with relative bandwidths of $10.6 \%$ and $12.1 \%$ at the lower and upper bands, respectively. As described in Section II the magnetic current distributions at both frequencies are similar, and therefore, the resulting radiation patterns are expected to be similar as well. The co- and cross-polarized E- and $\mathrm{H}$-plane radiation patterns of the antenna at both bands were measured, and the results are presented in Fig. 7(a) and (b). It is shown that the radiation patterns at both bands are similar and the levels of cross polarized radiation are negligible. The antenna gains are measured using a standard horn antenna and are reported in Table II.

\section{B. Parametric Study}

A similar parametric study as the one presented in Section II was performed for the antenna in the dual-band mode. In this study, the bandwidth and frequency ratio $\left(f_{u} / f_{l}\right)$ of the antenna in the dual-band mode is studied as functions of the aspect ratio, feed inpedance, and feed location. A number of different moderately-wide dual-band slot antennas with different $W / L, L_{s}$, and feed impedance values are designed and simulated in IE3D. Simultaneous matching at both bands to $50 \Omega$ is obtained by changing the length of open circuited microstrip line $\left(L_{m}\right.$ in Fig. 1). Fig. 8 shows the fractional bandwidth of the lower and upper bands $\left(f_{l}\right.$ and $\left.f_{u}\right)$ as funnctions of $W / L$ and $L_{s}$ for different values of feed impedances. For narrow microstrip feeds (high impedance lines) achieving simultaneous match at both bands was found to be easier than wider lines and lower $\left|S_{11}\right|$ values were obtained. As a result, the dual-band operation, for a fixed $L_{s}$, occurs at lower aspect ratios for narrower feeds. For example, for the $110 \Omega$ feed, the dual-band operation starts at $W / L=0.065$, which increases to $W / L=0.080$ and $W / L=0.095$ for $80 \Omega$ and $50 \Omega$ lines, respectively. However, one drawback of higher impedance feeds to lower ones is the slightly lower fractional bandwidths that are obtained as explained in Section II. The effects of $W / L$, feed impedance, and $L_{s}$ on the frequency ratio of the two bands of the dual-band antenna $\left(f_{u} / f_{l}\right)$ are also studied in Fig. 9. It is seen that increasing $L_{s}$ has the largest effect on $f_{u} / f_{l}$ and the aspect ratio of the antenna also affects it slightly, as shown in Fig. 9.

\section{An Octave Bandwidth Double-Element Slot ANTENNA DESIGN}

In this section the design and measurement results of a very wide-band, double-element slot antenna is presented. As demonstrated in Section II, a single-element, moderately-wide slot antenna can be designed to have a wide-band response. Therefore, it is expected that a properly designed antenna that is composed of two or more such radiators can show very wide-band characteristics. There are a number of different multi-element feeding topologies, in which such an element can be used. These include feeding the two elements in series, parallel, or through parasitic coupling. The wide-band behavior is only observed when the antenna is fed directly with a microstrip line; therefore, parasitic coupling does not provide the best results. Feeding both elements using a single microstrip line in series is possible and is a good choice for end fire arrays such as printed log-periodic arrays. However, using a parallel feed 
TABLE II

A Summary of the Physical and RAdiation PARAMETERs of the DuAL-BANd Slot ANTENNA OF SECTION III

\begin{tabular}{c||c|c|c|c|c}
\hline $\mathrm{L}, \mathrm{W}$ & $L_{s}, L_{m}$ & $f_{l}, f_{u}(G H z)$ & BW $@ f_{l}, f_{u}$ & Gain $@ f_{l}, f_{u}$ & $L_{g}, W_{g}$ \\
\hline $31 \mathrm{~mm}, 6 \mathrm{~mm}$ & $5 \mathrm{~mm}, 2.3 \mathrm{~mm}$ & $3.3,4.85$ & $10.6 \%, 12.1 \%$ & $2.5 \mathrm{~dB}, 2.8 \mathrm{~dB}$ & $15 \mathrm{~cm}, 12 \mathrm{~cm}$ \\
\hline
\end{tabular}

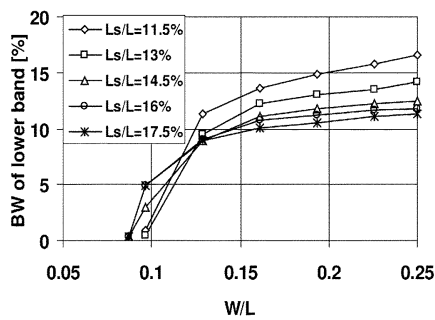

(a)

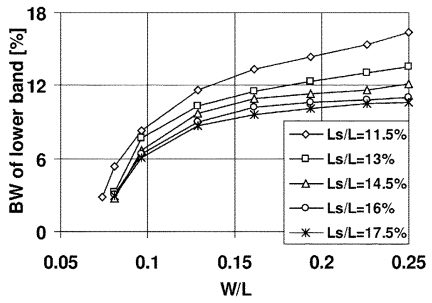

(c)

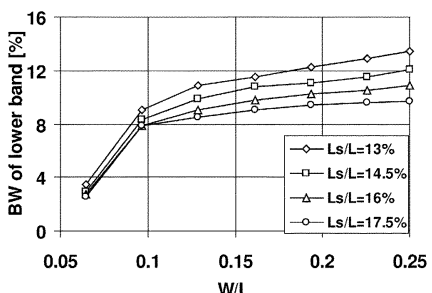

(e)

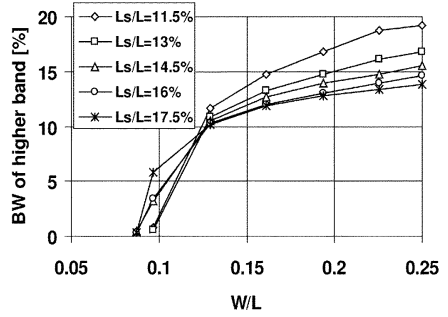

(b)

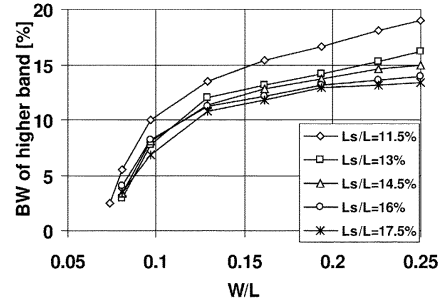

(d)

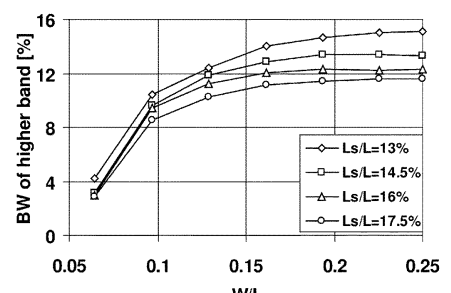

W/L

Fig. 8. Bandwidth of moderately-wide slot antennas in the dual-band mode of operation as a function of aspect ratio $(W / L)$, feed impedance, and location of the feed. (a) $50 \Omega$ feed impedance, lower band. (b) $50 \Omega$ feed impedance, higher band. (c) $80 \Omega$ feed impedance, lower band. (d) $80 \Omega$ feed impedance, higher band. (e) $110 \Omega$ feed impedance, lower band. (f) $110 \Omega$ feed impedance, higher band.

configuration, such as the one shown in Fig. 10(a), provides more flexibility in the design of the broad-band feed network and allows for designing antennas with a broadside radiation pattern. In this arrangement, a broad-band antenna with good impedance matching and consistent radiation patterns over the band of operation can easily be obtained.

The objective is to design an antenna that covers at least an octave bandwidth. For this design, the upper and lower frequencies are chosen such that they cover both of the bands designated for Wireless LAN operation. The schematic of the proposed antenna is shown in Fig. 10(a) where two slots with different lengths are used in a parallel feed arrangement. The larger antenna covers the lower and the smaller antenna covers the higher frequency bands. The lengths $L_{1}$ and $L_{2}$ are determined from the lowest resonant frequency of each element. $W_{1}$ and $W_{2}$ are chosen to be equal and wide enough such that the aspect ratio of both elements are in the range of $0.15 \leq W / L \leq 0.25$. From Fig. 4(c), it is seen that in this range, the antennas have their maximum bandwidth. Choosing $W_{2} / L_{2}=0.25$ results in $W_{2}=7 \mathrm{~mm}$. Although it is not necessary, $W_{1}$ is chosen

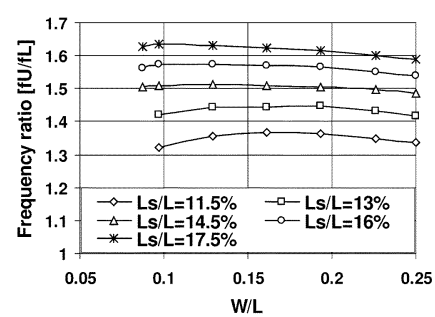

(a)

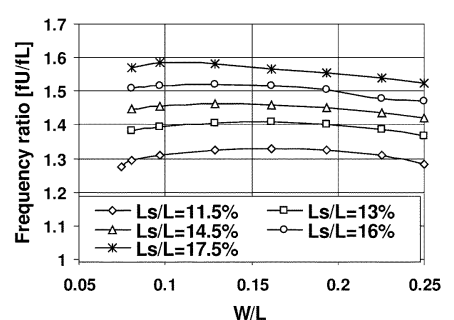

(b)

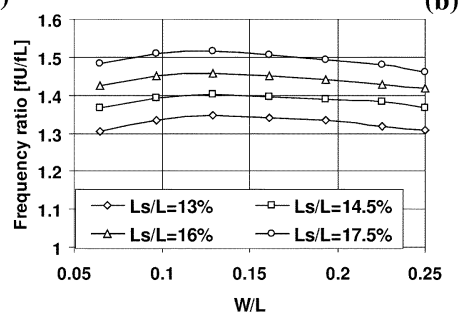

(c)

Fig. 9. Frequency ratios of the upper band to the lower band of the moderatelywide slot antenna in the dual-band mode of operation as functions of the aspect ratio $(W / L)$, feed impedance, and location of the feed. (a) $50 \Omega$ microstrip feed. (b) $80 \Omega$ microstrip feed. (c) $110 \Omega$ microstrip feed.

to be equal to $7 \mathrm{~mm}$ as well. This results in $W_{1} / L_{1}=0.175$, which is in the desired range. Then the larger slot antenna is simulated and $L_{S 1}$ is optimized such that the antenna covers a bandwidth of 2.3 to $3.6 \mathrm{GHz}$ and similarly, $L_{S 2}$ is optimized such that the second antenna covers a bandwidth of 3.7 to 5.5 GHz. The feed network dimensions $L_{3}, L_{4}, L_{5}, W_{3}, W_{4}, L_{m 1}$, and $L_{m 2}$ [see Fig. 10(a)], must be optimized to achieve the desired response in the band of operation. This is accomplished by performing a full-wave simulation on the four-port structure shown in Fig. 10(b) and obtaining the S-parameters of this network. Then, the calculated S-parameter matrix is used in ADS and a circuit simulation is performed on the circuit shown in Fig. 10(c). In this simulation, the software replaces all of the microstrip lines, the Tee-junction, and the microstrip taper by their equivalent circuit models. The values $L_{3}, L_{4}, L_{5}, W_{3}, W_{4}, L_{m 1}$, and $L_{m 2}$ are defined as optimization variables in this circuit simulation. The optimization goal is to have $\left|S_{11}\right|<-10 \mathrm{~dB}$ over the frequency range of 2.4 to $5.5 \mathrm{GHz}$. Two constraints are set on the variables; first the width of microstrip lines must be larger than $0.15 \mathrm{~mm}$. The reason for this is that the fabrication of very narrow microstrip lines is difficult in our facilities. The second constraint is that the difference between $L_{4}$ and $L_{5}$ [see Fig. 10(a)] must be smaller than $6 \mathrm{~mm}$. Since the electrical length of a $6 \mathrm{~mm}$ microstrip line at $5.5 \mathrm{GHz}$ (which is the highest desired frequency) on the RO4350B substrate is about $60^{\circ}$, the two antennas will not be excited with a phase difference larger than $60^{\circ}$ at any frequency in the desired band of operation. Even though each antenna covers a certain portion of the band, there is an overlap frequency band in which both of the antennas can operate. Therefore, the second constraint is 


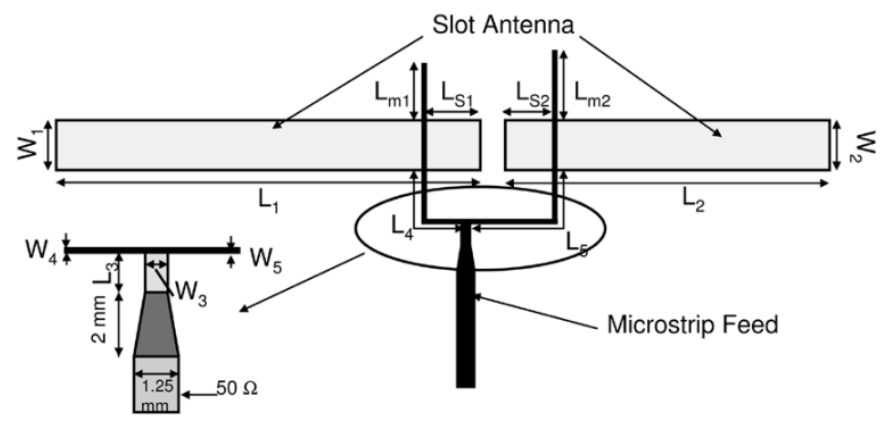

(a)

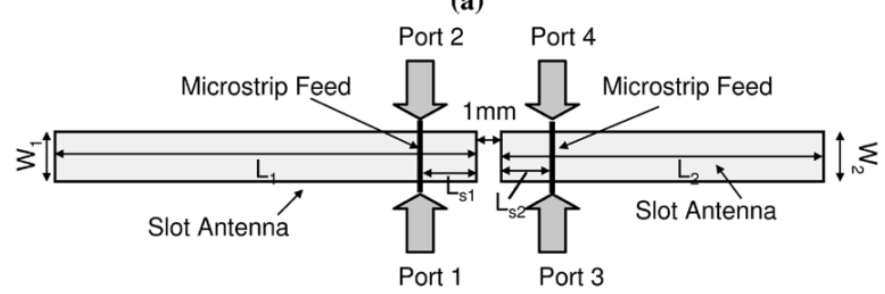

(b)

Four-port network containing the $S$ parameters of the antennas in the arrangement of Figure 10(b)

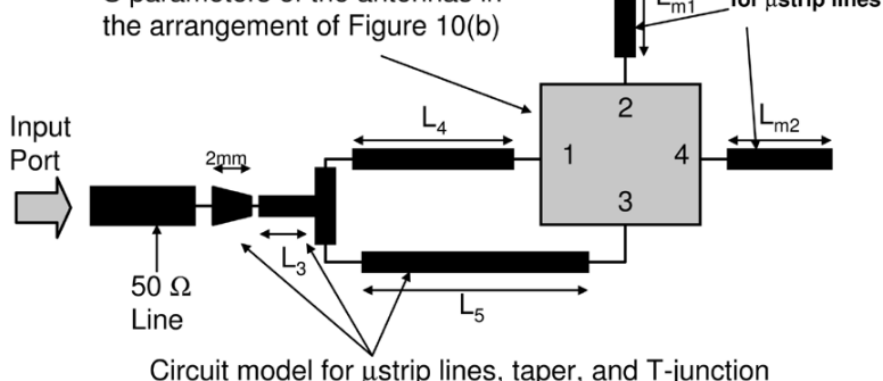

(c)

Fig. 10. Geometry and circuit model of the double-element broad-band slot antenna of Section IV. (a) Antenna geometry. (b) The four-port network, which models the antennas, their coupling effects, and their microstrip feeds. The S-parameters of this network are used in the simulation of the circuit shown in (c). (c) Circuit model of the antenna in (a) used in optimizing the feed network parameters.

TABLE III

A Summary of the Physical Dimensions of THE BROADBAND DOUBle-ElEMENT ANTENNA OF SECTION IV. ALl Dimensions ARE IN MILLIMETERS

\begin{tabular}{c||c|c|c|c|c|c|c}
\hline Parameter & $L_{1}$ & $L_{2}$ & $L_{s 1}$ & $L_{s 2}$ & $L_{m l}$ & $L_{m 2}$ & $L_{3}$ \\
\hline Value & 40 & 28 & 6.1 & 4.6 & 3.5 & 4.6 & 1.25 \\
\hline Parameter & $L_{4}$ & $L_{5}$ & $W_{1}$ & $W_{2}$ & $W_{3}$ & $W_{4}$ & $W_{5}$ \\
\hline Value & 7.3 & 12.6 & 7 & 7 & 0.7 & 0.2 & 0.2 \\
\hline
\end{tabular}

necessary for having consistent radiation patterns over the entire frequency band. The circuit was then optimized using the embedded optimization engine in ADS [10] and the optimum values of the parameters were obtained. Since the circuit simulation and optimization process ignores mutual coupling effects between the microstrip lines, a full-wave simulation was performed on the entire structure and the optimization parameters were fine tuned in the full-wave simulation. For the aforementioned frequency band, the final physical parameters of the antenna are listed in Table III.

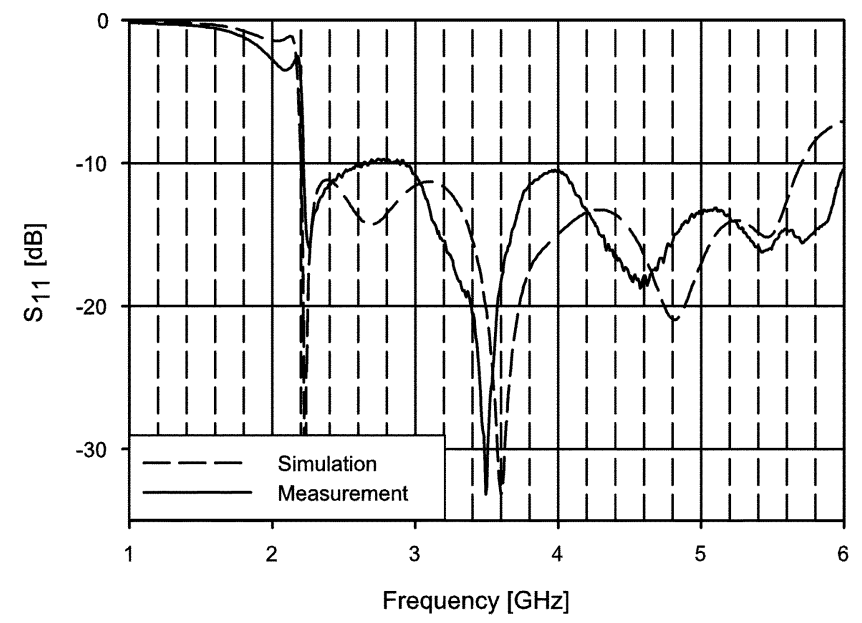

Fig. 11. Simulated and measured input reflection coefficient $\left(s_{11}\right)$ of the double-element broad-band antenna of Section IV.

The final design was fabricated on a RO4350B substrate with a ground plane size of $15 \mathrm{~cm} \times 12 \mathrm{~cm}$. The $S_{11}$ of the antenna was measured using a calibrated HP-8753D vector network analyzer and is presented in Fig. 11 along with the full-wave simulation result obtained from IE3D. The discrepancies between the two results can mostly be attributed to the finite size of the ground plane, inaccuracies in the fabrication process, uncertainties in the exact permittivity of the substrate $( \pm 3 \%$ error specified by the manufacturer), and numerical errors in the simulation results. Nevertheless, the measured $S_{11}$ shows a $-10 \mathrm{~dB}$ impedance bandwidth from 2.3 to $6 \mathrm{GHz}$. However, in order to determine the useful bandwidth of the antenna, other parameters such as radiation patterns, gain, polarization, and cross polarization levels must also be carefully examined over the entire bandwidth.

In order to study the effect of ground plane size on the radiation patterns of the wide-band antenna, another antenna with the same dimensions was also fabricated on a substrate with a larger ground plane $(23 \mathrm{~cm} \times 30 \mathrm{~cm})$. The radiation patterns of both antennas were measured in the anechoic chamber at nine different frequencies ranging from 2.5 to $6 \mathrm{GHz}$, but for brevity, only the radiation patterns at $3,4,5$, and $5.8 \mathrm{GHz}$ are reported. Figs. 12 and 13 show the $\mathrm{H}$ - and E-plane radiation patterns for both co- and cross-polarizations at different frequencies. In these figures SGP and LGP denote "small ground plane" and "large ground plane," respectively. The experimental results show that the size of the ground plane alters the radiation pattern, especially in the $\mathrm{H}$-plane cut due to the out of phase radiation from the edges of the substrate.

The measured gain and directivity of the antenna at boresight are shown in Fig. 14. Also the directivity at the direction of maximum radiation is shown in this figure. The antenna gain and directivity at boresight are very close to each other, indicating a high radiation efficiency. This figure also shows that above $5.5 \mathrm{GHz}$ the direction of maximum radiation shifts away from the boresight direction. There are two reasons for this: First, at $5.5 \mathrm{GHz}$ the larger antenna has an electrical length that is about $1 \lambda$. Since it is off-centered fed, its electric field distribution is 

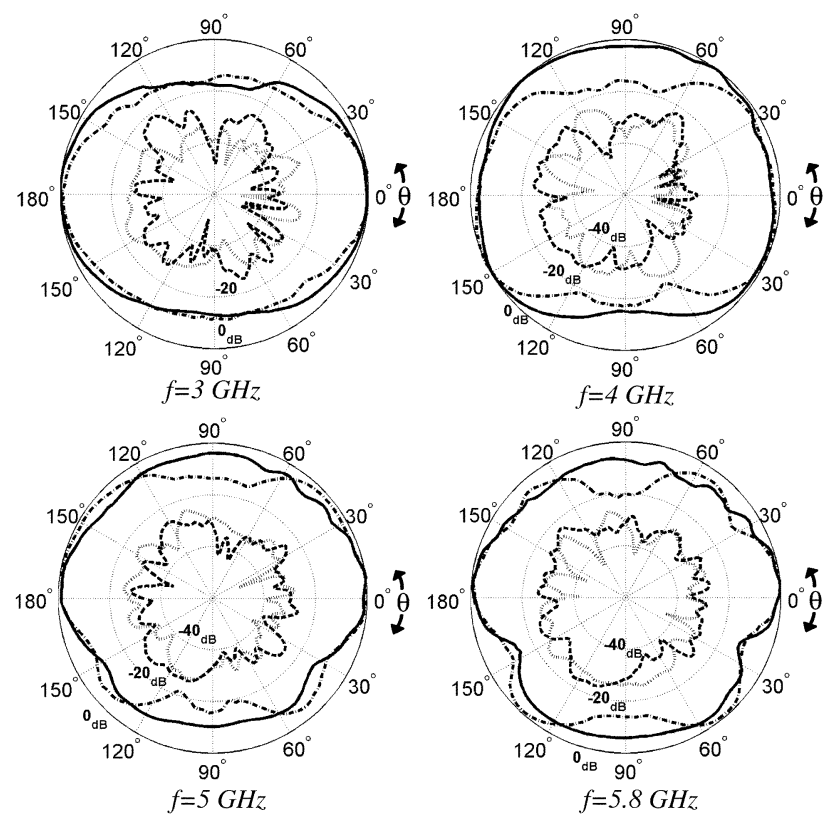

Fig. 12. H-plane co- and cross-polarized radiation patterns of the doubleelement broad-band antenna of Section IV. "-": H-plane co-pol SGP, “-.": H-plane co-pol LGP, “- -”: H-plane X-pol SGP, and “...”: H-plane X-pol LGP.
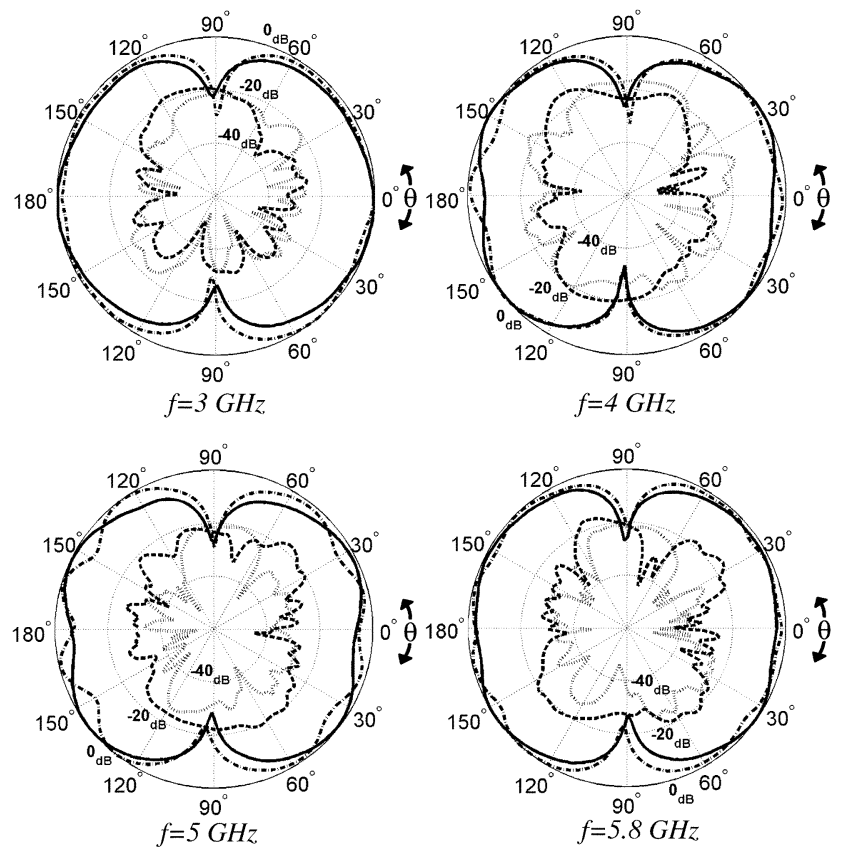

Fig. 13. E-plane co- and cross-polarized radiation patterns of the doubleelement broad-band antenna of Section IV. "-" solid line: E-plane co-pol SGP, “-.” Dash-dotted line: E-plane co-pol LGP, “. -." dash-dashed line: E-plane X-pol SGP, and “...” dotted line: E-plane X-pol LGP.

anti-symmetric with respect to the center of the antenna. Therefore, there is a null in the radiation pattern of this element at boresight and the maximum radiation occurs at $\theta=45^{\circ}, 135^{\circ}$. Since this element is resonant at around $1 \lambda$, it can radiate rather efficiently and distort the overall radiation pattern. The second reason is that, as frequency increases, the two antennas are excited with a larger phase difference, which also shifts the direction of maximum radiation. However, this happens outside of

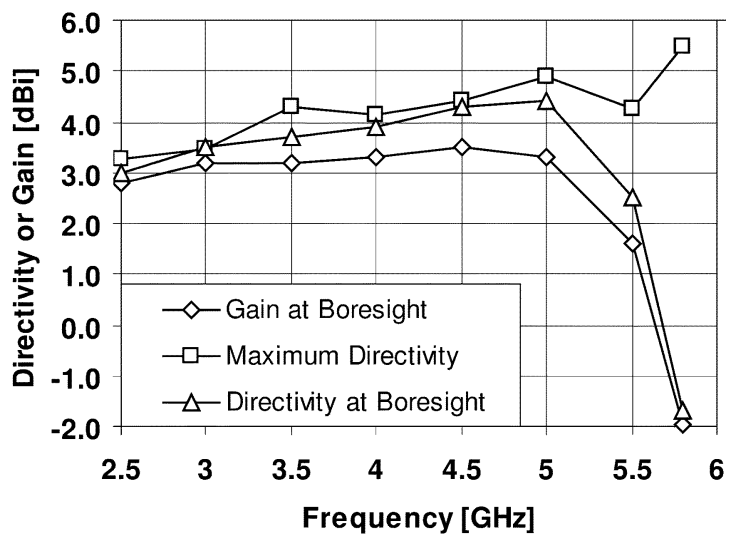

Fig. 14. Measured values of the gain and computed values of the directivity of the double-element broad-band antenna.

the desired band of operation. Based on the measured $S_{11}$, consistency of the radiation patterns, and polarization purity over the entire frequency band, it can be concluded that the antenna has a bandwidth from 2.3 to $5.8 \mathrm{GHz}$ or equivalently a $2.5: 1$ bandwidth ratio.

\section{CONCLUSION}

A novel slot antenna element is proposed that allows for easy design of wide-band or dual-band antennas. The single element shows bandwidths up to $37 \%$ when used in the wide-band mode, and when operated in a dual-band mode, the structure has the ability to cover a 1.6:1 frequency ratio easily. Furthermore it is shown that this broad-band antenna element is an excellent choice for constructing multi-element antennas with a much larger bandwidth. To demonstrate this, a two-element antenna configuration was considered and a wide-band broadside radiating antenna with a bandwidth ratio of 2.5:1 was designed. This allows the designer to obtain very wide-band antennas by using a smaller number of antenna elements than was previously possible. An important and unique feature of this design is that the polarization purity of the antenna and high efficiency are unchanged over the entire frequency band.

\section{ACKNOWLEDGMENT}

The authors would like to thank the anonymous reviewers for their constructive comments.

\section{REFERENCES}

[1] M. Kahrizi, T. Sarkar, and Z. Maricevic, "Analysis of a wide radiating slot in the ground plane of a microstrip line," IEEE Trans. Microwave Theory Tech., vol. 41, pp. 29-37, Jan. 1993.

[2] J. Sze and K. Wong, "Bandwidth enhancement of a microstrip-line-fed printed wide-slot antenna," IEEE Trans. Antennas Propag., vol. 49, pp. 1020-1024, Jul. 2001.

[3] H. L. Lee, H. J. Lee, J. G. Yook, and H. K. Park, "Broadband planar antenna having round corner rectangular wide slot," in Proc. IEEE Antennas and Propagation Society Int. Symp., vol. 2, Jun. 2002, pp. 460-463.

[4] P. H. Rao, V. Fusco, and R. Cahill, "A broadband antenna for PCN/ UMTS applications," in Proc. High Frequency Postgraduate Student Colloquium, Sep. 2000, pp. 2-5. 
[5] X. Liang and C. Y. W. Michael, "A microstrip-fed wide-band slot array with finite ground," in Proc. IEEE Antennas and Propagation Society Int. Symp., vol. 2, Jul. 2000, pp. 510-513.

[6] D. Llorens, P. Otero, and C. Camacho-Penalosa, "Dual-band single CPW port planar-slot antenna," IEEE Trans. Antennas Propag., vol. 51, pp. 137-139, Jan. 2003.

[7] C. A. Balanis, Antenna Theory: Analysis and Design, 2nd ed. New York: Wiley, 1996, ch. 4, sec. 4.8.

[8] "IE3D Electromagnetic Simulation and Optimization Software," Zeland Software, Inc.

[9] N. Behdad and K. Sarabandi, "Double resonant slot antennas with enhance bandwidth," in Proc. IEEE APS/URSI Symp., Monterey, CA, Jun. 22-26, 2004.

[10] "Advance Design Systems," Agilent Technologies, Palo Alto, CA 94306.

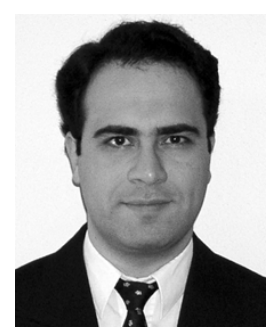

Nader Behdad (S'98) was born in Mashhad, Iran, in 1977. He received the B.Sc. degree from Sharif University of Technology, Tehran, Iran, in 2000 and the M.Sc. degree from the University of Michigan, Ann Arbor, MI, in 2003, where he is currently working toward the Ph.D. degree in the Department of Electrical Engineering and Computer Science, with a focus on bandwidth enhancement and miniaturization of printed antennas.

From 2000 to 2001, he was with the Electronics Research Center, Sharif Unviersity of Technology, as an Antenna Design Engineer working on the design of antennas for wireless local loop (WLL) systems. Since January 2002, he has been working as a Research Assistant in the Radiation Laboratory, University of Michigan.

Mr. Behdad is the recipient of the Best Student Paper Award in the Antenna Applications Symposium held in Monticelo, IL, in September 2003 and was the winner of the second prize in the student paper competition of the USNC/URSI National Radio Science Meeting, Boulder, CO, in January 2004.

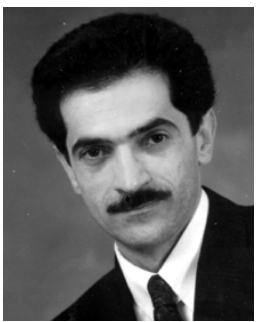

Kamal Sarabandi (S'87-M'90-SM'92-F'00) received the B.S. degree in electrical engineering from Sharif University of Technology, Tehran, Iran, in 1980, the M.S. degree in electrical engineering/ mathematics, and the Ph.D. degree in electrical engineering from The University of Michigan-Ann Arbor, in 1986 and 1989, respectively.

$\mathrm{He}$ is Director of the Radiation Laboratory and a Professor in the Department of Electrical Engineering and Computer Science, The University of Michigan-Ann Arbor. He has 20 years of experience with wave propagation in random media, communication channel modeling, microwave sensors, and radar systems and is leading a large research group consisting of four research scientists, ten Ph.D. students, and two M.S. students. Over the past ten years he has graduated $20 \mathrm{Ph}$.D. students. He has served as the Principal Investigator on many projects sponsored by NASA, JPL, ARO, ONR, ARL, NSF, DARPA and numerous industries. He has published many book chapters and more than 115 papers in refereed journals on electromagnetic scattering, random media modeling, wave propagation, antennas, microwave measurement techniques, radar calibration, inverse scattering problems, and microwave sensors. He has also had more than 230 papers and invited presentations in many national and international conferences and symposia on similar subjects. His research areas of interest include microwave and millimeter-wave radar remote sensing, electromagnetic wave propagation, and antenna miniaturization.

Dr. Sarabandi is a Member of the International Scientific Radio Union (URSI) Commission $\mathrm{F}$ and of The Electromagnetic Academy. He received the Henry Russel Award from the Regent of The University of Michigan-Ann Arbor (the highest honor the University of Michigan bestows on a faculty member at the assistant or associate level). He received a 1996 Teaching Excellence Award from the Department of Electrical Engineering and Computer Science and a 1999 GAAC Distinguished Lecturer Award from the German Federal Ministry for Education, Science, and Technology, given to about ten individuals worldwide in all areas of engineering, science, medicine, and law. He also received the 2003/2004 College of Engineering Research Excellence Award, The University of Michigan-Ann Arbor. In the past several years, joint papers presented by his students at a number of symposia (IEEE AP'95,'97,'00,'01,'03 IEEE IGARSS'99,'02, IEEE MTTS'01) have received student prize paper awards. He is a Vice President of the IEEE Geoscience and Remote Sensing Society (GRSS), a past Chairman of the Awards Committee of the IEEE GRSS from 1998 to 2002, and a Member of the IEEE Technical Activities Board Awards Committee from 2000 to 2002. He is an Associate Editor of the IEEE TRANSACTIONS ON ANTENNAS AND PROPAGATION and the IEEE SENSORS JouRnal. He is listed in American Men \& Women of Science, Who's Who in America, and Who's Who in Electromagnetics. 